\title{
A CHARACTERIZATION OF SUBMETACOMPACTNESS IN TERMS OF PRODUCTS
}

\author{
YUKINOBU YAJIMA
}

(Communicated by Dennis Burke)

\begin{abstract}
A space $X$ is said to be suborthocompact if for every open cover $\mathscr{U}$ of $X$ there is a sequence $\left\{\mathscr{V}_{n}\right\}$ of open refinements of $\mathscr{U}$ such that for each $x \in X$ there is some $n \in \omega$ such that $\bigcap\left\{V \in \mathscr{V}_{n}: x \in V\right\}$ is a neighborhood of $x$ in $X$. It is proved that a Tychonoff space $X$ is submetacompact if and only if the product $X \times \beta X$ is suborthocompact.
\end{abstract}

\section{INTRODUCTION}

According to Tamano's Theorem [T1], a Tychonoff space $X$ is paracompact if and only if the product $X \times \beta X$ is normal, where $\beta X$ denotes the Stone-Čech compactification of $X$. As a nice analogue, Junnila [Ju1] proved that a Tychonoff space $X$ is metacompact if and only if the product $X \times \beta X$ is orthocompact. Our purpose in this paper is to give another analogue for submetacompactness.

Main Theorem. A Tychonoff space $X$ is submetacompact if and only if the product $X \times \beta X$ is suborthocompact.

In $\S 1$, we will precisely introduce the concept of suborthocompact spaces, whose class contains the classes of submetacompact spaces and orthocompact spaces. In $\S 2$, our Main Theorem will be proved in a slightly generalized form. Second, we consider Katuta-Junnila's problem, which was first raised in [K, Problems 2.6 and 2.7]. Jiang [J] gave a partial answer to this problem. In $\S 3$, we will give an extension of his result, using suborthocompactness.

All spaces dealt with here are topological spaces. No separation axioms are assumed, unless otherwise stated.

\section{SUBMetACOMPACTNESS AND SUBORTHOCOMPACTNESS}

Let $X$ be a space. For two covers $\mathscr{U}$ and $\mathscr{V}$ of $X, \mathscr{V}$ is a refinement of $\mathscr{U}$ (or $\mathscr{V}$ refines $\mathscr{U}$ ) if each member of $\mathscr{V}$ is contained in some member of

Received by the editors November 28, 1989.

1980 Mathematics Subject Classification (1985 Revision). Primary 54D18; Secondary 54B10.

Key words and phrases. Submetacompact, suborthocompact, product, metacompact, directed cover, cushioned refinement. 
$\mathscr{U}$. For a subset $Y$ of $X, \mathrm{Cl} Y$ or $\mathrm{Cl}_{X} Y$ denotes the closure of $Y$ in $X$. Let $\mathscr{V}$ be a collection of subsets of a space $X$. For each $x \in X$, let

$$
\begin{aligned}
\mathscr{V}(x) & =\{V \in \mathscr{V}: x \in V\}, \\
\bigcap \mathscr{V}(x) & =\bigcap\{V \in \mathscr{V}: x \in V\},
\end{aligned}
$$

and

$$
\operatorname{St}(x, \mathscr{V})=\bigcup\{V \in \mathscr{V}: x \in V\}=\bigcup \mathscr{V}(x) .
$$

A space $X$ is submetacompact (or $\theta$-refinable) if for every open cover $\mathscr{U}$ of $X$ there is a sequence $\left\{\mathscr{V}_{n}\right\}$ of open refinements of $\mathscr{U}$ such that, for each $x \in X$, there is some $n \in \omega$ such that $\mathscr{V}_{n}(x)$ is finite (i.e., $\mathscr{V}_{n}$ is point-finite at $x$ ).

However, we will prefer to make use of the following characterization of submetacompactness.

Theorem 1.1 (Junnila [Ju2]). A space $X$ is submetacompact if and only if, for every open cover $\mathscr{U}$ of $X$, there is a sequence $\left\{\mathscr{W}_{n}\right\}$ of open covers of $X$ such that, for each $x \in X$, there are an $n \in \omega$ and a finite subcollection $\mathscr{U}_{x}$ of $\mathscr{U}$ such that $\operatorname{St}\left(x, \mathscr{W}_{n}\right) \subset \cup \mathscr{U}_{x}$ and $x \in \cap \mathscr{U}_{x}$.

A space $X$ is orthocompact if every open cover $\mathscr{U}$ of $X$ has an open refinement $\mathscr{V}$ such that $\cap \mathscr{V}(x)$ is open in $X$ for each $x \in X$ (i.e., $\mathscr{V}$ is interior-preserving).

Now, we introduce the following concept:

Definition 1.2. A space $X$ is said to be suborthocompact if, for every open cover $\mathscr{U}$ of $X$, there is a sequence $\left\{\mathscr{V}_{n}\right\}$ of open refinements of $\mathscr{U}$ such that for each $x \in X$ there is some $n \in \omega$ such that $\cap \mathscr{V}_{n}(x)$ is a neighborhood of $x$ in $X$.

It is clear that both submetacompact spaces and orthocompact spaces are suborthocompact. Note that each closed subspace of a suborthocompact space is suborthocompact.

Remark 1.3. Submetacompactness is preserved under closed maps (cf. [Ju2]). However, orthocompactness is not preserved under perfect maps. In fact, Burke [B1] gave an example of an orthocompact space $X$ and a perfect map $f: X \rightarrow Y$ onto a nonorthocompact space $Y$. Seeing his proof for the nonorthocompactness of $Y$, it is easy to observe that the space $Y$ is not suborthocompact. Hence suborthocompactness is not preserved under perfect maps.

\section{Generalized Main Theorem}

Let us start with the following lemma, which is easy to verify (e.g., see [B2, Theorem 6.1]). 
Lemma 2.1. If $X$ is a submetacompact space and $C$ is a compact Hausdorff space, then the product $X \times C$ is submetacompact.

It has been proved in [GY] that the above "compact Hausdorff space" can be extended to "regular space with a $\sigma$-closure-preserving cover by compact sets."

Let $X$ and $Y$ be spaces. For a collection $\mathscr{H}$ of subsets of the product $X \times Y$ and an $(x, y) \in X \times Y$, let $\mathscr{H}(x, y)=\{H \in \mathscr{H}:(x, y) \in H\}$. A subset $R$ of $X \times Y$ is an open rectangle if it is an open set of the form $A \times B$ (i.e., $R=A \times B$ such that $A$ and $B$ are open in $X$ and $Y$, respectively).

In order to prove our Main Theorem, we are to prove the following generalized result, which is an analogue of [T2, Theorem 3.1], [M1, Theorem 2.7], and [Ju1, Theorem 4.1].

Theorem 2.2. Let $\gamma X$ be a compactification of a Tychonoff space $X$. Then $X$ is submetacompact if and only if the product $X \times \gamma X$ is suborthocompact.

Proof. Since the "only if" part is an immediate consequence of Lemma 2.1, we prove only the "if" part.

Let $\mathscr{U}$ be any open cover of $X$. Take an open set $U^{*}$ in $\gamma X$ with $U^{*} \cap X=$ $U$ for each $U \in \mathscr{U}$. Let $\mathscr{U}^{*}=\left\{U^{*}: U \in \mathscr{U}\right\}$. Take a collection $\mathscr{V}$ of open sets in $\gamma X$ such that $\bigcup \mathscr{V}=\bigcup \mathscr{U}^{*}$ and, for each $V \in \mathscr{V}$, one can find $U_{V} \in \mathscr{U}$ with $\mathrm{Cl}_{\gamma X} V \subset U_{V}^{*}$. Let

$$
\mathscr{G}=\left\{(V \cap X) \times U_{V}^{*}: V \in \mathscr{V}\right\} \cup\left\{(V \cap X) \times\left(\gamma X \backslash \mathrm{Cl}_{\gamma X} V\right): V \in \mathscr{V}\right\}
$$

Then $\mathscr{G}$ is an open cover of $X \times \gamma X$. There is a sequence $\left\{\mathscr{H}_{n}\right\}$ of open refinements of $\mathscr{G}$ such that, for each $\left(x, x^{\prime}\right) \in X \times \gamma X$, one can choose $n \in \omega$ such that $\cap \mathscr{H}_{n}\left(x, x^{\prime}\right)$ is a neighborhood of $\left(x, x^{\prime}\right)$ in $X \times \gamma X$. For each $\left(x, x^{\prime}\right) \in X \times \gamma X$ and each $n \in \omega$, we take an open rectangle $P_{x, x^{\prime}, n} \times Q_{x, x^{\prime}, n}$ in $X \times \gamma X$, that contains the point $\left(x, x^{\prime}\right)$ and is contained in some member of $\mathscr{H}_{n}$. Since $\gamma X$ is compact, for each $x \in X$ and each $n \in \omega$, there are an open neighborhood $W_{x, n}$ of $x$ in $X$ and a finite subset $F(x, n)$ of $\gamma X$ such that

(i) $W_{x, n} \subset \bigcap_{z \in F(x, n)} P_{x, z, n}$,

(ii) $\gamma X=\bigcup_{z \in F(x, n)} Q_{x, z, n}$, and

(iii) $W_{x, n+1} \subset W_{x, n}$.

Here, we set $\mathscr{W}_{n}=\left\{W_{x, n}: x \in X\right\}$ for each $n \in \omega$. We show that the sequence $\left\{\mathscr{W}_{n}\right\}$ of open covers of $X$ satisfies the condition of Theorem 1.1. For this, we need to prove the following claim, corresponding to a similar statement in the proof of [B2, Theorem 2.10].

Claim. For each $x \in X$, there is some $k \in \omega$ such that $\mathrm{Cl}_{\gamma X} \operatorname{St}\left(x, \mathscr{W}_{k}\right) \subset$ $\operatorname{St}\left(x, \mathscr{U}^{*}\right)$.

Proof. Assume that we can pick some $x \in X$ such that

$$
\mathrm{Cl}_{\gamma X} \operatorname{St}\left(x, \mathscr{W}_{n}\right) \backslash \mathrm{St}\left(x, \mathscr{U}^{*}\right) \neq \varnothing
$$


for each $n \in \omega$. By (iii), $\mathrm{Cl}_{\gamma X} \operatorname{St}\left(x, \mathscr{W}_{n+1}\right) \subset \mathrm{Cl}_{\gamma X} \operatorname{St}\left(x, \mathscr{W}_{n}\right)$ for each $n \in \omega$. So there is some $q \in \bigcap_{n \in \omega} \mathrm{Cl}_{\gamma X} \operatorname{St}\left(x, \mathscr{W}_{n}\right) \backslash \mathrm{St}\left(x, \mathscr{U}^{*}\right)$. Choose $k \in \omega$ such that $\cap \mathscr{H}_{k}(x, q)$ is a neighborhood of $(x, q)$ in $X \times \gamma X$. Take an open rectangle $S \times T$ in $X \times \gamma X$ such that $(x, q) \in S \times T \subset \cap \mathscr{H}_{k}(x, q)$. We can pick $p \in X$ such that $p \in T \cap \operatorname{St}\left(x, \mathscr{W}_{k}\right)$, and we can pick $y \in X$ such that $x \in W_{y, k}$ and $p \in W_{y, k}$. By (ii), pick $z \in F(y, k)$ with $q \in Q_{y, z, k}$. Then it follows from (i) that

$$
\{(x, q),(p, q)\} \subset W_{y, k} \times Q_{y, z, k} \subset P_{y, z, k} \times Q_{y, z, k} \subset H_{0},
$$

for some $H_{0} \in \mathscr{H}_{k}$. By $H_{0} \in \mathscr{H}_{k}(x, q)$, we have

$$
(x, p) \in S \times T \subset \bigcap \mathscr{\mathscr { H }}_{k}(x, q) \subset H_{0} .
$$

Hence we obtain the result that $\{(x, q),(x, p),(p, q)\} \subset H_{0} \in \mathscr{H}_{k}$. By $q \notin$ $\operatorname{St}\left(x, \mathscr{U}^{*}\right)$, note that $(x, q) \notin(V \cap X) \times U_{V}^{*}$ for each $V \in \mathscr{V}$. Since $\mathscr{H}_{k}$ refines $\mathscr{G}$, there is a $V_{0} \in \mathscr{V}$ such that $H_{0} \subset\left(V_{0} \cap X\right) \times\left(\gamma X \backslash \mathrm{Cl}_{\gamma X} V_{0}\right)$. Therefore, we have

$$
\{(x, p),(p, q)\} \subset\left(V_{0} \cap X\right) \times\left(\gamma X \backslash \mathrm{Cl}_{\gamma X} V_{0}\right) .
$$

This is a contradiction. Our claim has been proved.

Pick any $x \in X$. By the claim, choose some $k \in \omega$ such that $\mathrm{Cl}_{\gamma X} \operatorname{St}\left(x, \mathscr{W}_{k}\right)$ $\subset \operatorname{St}\left(x, \mathscr{U}^{*}\right)$. There is a finite subcollection $\mathscr{U}_{x}=\left\{U_{1}, \ldots, U_{m}\right\}$ of $\mathscr{U}$ such that $\mathrm{Cl}_{\gamma X} \operatorname{St}\left(x, \mathscr{W}_{k}\right) \subset \bigcup_{i \leq m} U_{i}^{*}$ and $x \in \bigcap_{i \leq m} U_{i}^{*}$. Then we have $\operatorname{St}\left(x, \mathscr{W}_{k}\right) \subset$ $\cup \mathscr{U}_{x}$ and $x \in \cap \mathscr{U}_{x}$. Thus it follows from Theorem 1.1 that $X$ is submetacompact. The proof is complete.

Remark 2.3. In Theorem 2.2, a compactification $\gamma X$ of $X$ can be replaced by a compact Hausdroff space $K$ which contains $X$ as a subspace, since suborthocompactness is inherited by closed subspaces.

For a space $X, L(X)$ denotes the Lindelöf number of $X$; i.e.,

$$
\begin{array}{r}
L(X)=\aleph_{0} \cdot \min \{\mathbf{m}: \text { every open cover of } X \text { has a subcover } \\
\text { of cardinality } \leq \mathbf{m}\} .
\end{array}
$$

For a cardinality $\mathbf{m}, 2^{\mathbf{m}}$ denotes the product of $\mathbf{m}$ copies of the discrete twopoint space.

Morita [M2] actually showed that a Hausdroff space $X$ is paracompact if and only if the product $X \times 2^{L(X)}$ is normal. As an analogue, Scott [S] showed that a space $X$ is metacompact if and only if the product $X \times 2^{L(X)}$ is orthocompact. Here, we can also obtain the following analogue:

Theorem 2.4. A space $X$ is submetacompact if and only if the product $X \times 2^{L(X)}$ is suborthocompact. 
However, the proof is similar to that of [S, Lemma 1.6]. The details of it are left to the reader.

\section{KATUtA-JUNNILA'S PROBLEM}

A cover $\mathscr{V}$ of a space $X$ is directed if, for each $V_{0}, V_{1} \in \mathscr{V}$, there is some $V_{2} \in \mathscr{V}$ such that $V_{0} \cup V_{1} \subset V_{2}$.

This problem, which was raised in [Ju2 and $\mathrm{K}$ ], asks whether a space $X$ is metacompact (submetacompact) if every directed open cover of $X$ has a $(\sigma-)$ cushioned refinement. Jiang $[\mathrm{J}]$ gave an affirmative answer to the metacompact case assuming that the space $X$ is orthocompact. We are to generalize this result, using the suborthocompactness of $X$.

Theorem 3.1. A space $X$ is metacompact if and only if $X$ is suborthocompact and every directed open cover of $X$ has a cushioned refinement.

Proof. Since the "only if" part is obvious from [Ju1, Theorem 3.1], we show only the "if" part.

Let $\mathscr{U}$ be an open cover of $X$. There is a sequence $\left\{\mathscr{V}_{n}\right\}$ of open refinements of $\mathscr{U}$ such that, for each $x \in X$, one can choose $n \in \omega$ such that $\cap \mathscr{V}_{n}(x)$ is a neighborhood of $x$ in $X$. Take an $n \in \omega$. Let

$$
X_{n}=\left\{x \in X: \bigcap \mathscr{V}_{n}(x) \text { is a neighborhood of } x \text { in } X\right\},
$$

and let $\mathscr{V}_{n}^{F}$ be the open cover of $X$ which consists of all finite unions of members of $\mathscr{V}_{n}$. Since $\mathscr{V}_{n}^{F}$ is directed, it has a cushioned refinement $\mathscr{C}_{n}$. So $\mathscr{V}_{n}^{F}$ has a subcover $\left\{V^{*}(C): C \in \mathscr{C}_{n}\right\}$ such that $\operatorname{Cl}(\bigcup\{C: C \in \mathscr{A}\}) \subset$ $\bigcup\left\{V^{*}(C): C \in \mathscr{A}\right\}$ for each $\mathscr{A} \subset \mathscr{C}_{n}$. For each $x \in X_{n}$, let

$$
W_{x, n}=\operatorname{Int}\left(\bigcap \mathscr{V}_{n}(x)\right) \backslash \mathrm{Cl}\left(\bigcup\left\{C \in \mathscr{C}_{n}: x \notin V^{*}(C)\right\}\right) \text {. }
$$

Then each $W_{x, n}$ is an open neighborhood of $x$ in $X$.

Let $G_{n}=\bigcup\left\{W_{x, n}: x \in X_{n}\right\}$. Since every countable, increasing open cover of $X$ is directed, it has a cushioned refinement. So it follows from [I, Corollary] that $X$ is countably metacompact. Since $\left\{G_{n}: n \in \omega\right\}$ is a countable open cover of $X$, there is a point-finite open cover $\left\{H_{n}: n \in \omega\right\}$ of $X$ such that $H_{n} \subset G_{n}$ for each $n \in \omega$. Here, we set

$$
\mathscr{W}=\left\{W_{x, n} \cap H_{n}: x \in X_{n} \text { and } n \in \omega\right\} .
$$

Then $\mathscr{W}$ is an open refinement of $\mathscr{U}$. We show that $\mathscr{W}$ is a pointwise $W$ refinement of $\mathscr{U}$ (that is, for each $x \in X$, there is a finite subcollection $\mathscr{U}_{x}$ of $\mathscr{U}$ such that each member of $\mathscr{W}(x)$ is contained in some member of $\left.\mathscr{U}_{x}\right)$.

Pick $x \in X$. Let $N_{x}=\left\{n \in \omega: x \in H_{n}\right\}$. For each $n \in \omega$, we choose a $C_{n} \in \mathscr{C}_{n}$ and a finite subcollection $\mathscr{V}_{n, x}$ of $\mathscr{V}_{n}$ such that $x \in C_{n}$ and $V^{*}\left(C_{n}\right)=\bigcup \mathscr{V}_{n, x}$. We set $\mathscr{V}_{x}=\bigcup\left\{\mathscr{V}_{n, x}: n \in N_{x}\right\}$. Since $N_{x}$ is finite, so is $\mathscr{V}_{x}$. Since each $\mathscr{V}_{n}$ refines $\mathscr{U}^{x}$, it suffices to show that each member of $\mathscr{W}(x)$ is contained in some member of $\mathscr{V}_{x}$. Assume that $x \in W_{y, n} \cap H_{n}$, where 
$y \in X_{n}$. Then $n$ is in $N_{x}$. If $y \notin V^{*}\left(C_{n}\right)$, then we have $W_{y, n} \cap C_{n}=\varnothing$, which contradicts $x \in C_{n}$. Hence we get $y \in V^{*}\left(C_{n}\right)=\bigcup \mathscr{V}_{n, x}$. Find some $V_{0} \in \mathscr{V}_{n, x} \subset \mathscr{V}_{x}$ with $y \in V_{0}$. Then we have $W_{y, n} \subset \bigcap \mathscr{V}_{n}(y) \subset V_{0}$. Hence $\mathscr{W}$ is a pointwise $W$-refinement of $\mathscr{U}$. It follows from [W, Theorem 1] that $X$ is metacompact. The proof is complete.

However, we cannot obtain an analogue of Theorem 3.1 for submetacompactness. So we raise it as a problem:

Problem 3.2. If every directed open cover of a suborthocompact space $X$ has a $\sigma$-cushioned refinement, is $X$ then submetacompact?

\section{REFERENCES}

[B1] D. K. Burke, Orthocompactness and perfect maps, Proc. Amer. Math. Soc. 79 (1980), 484486.

[B2] _ Covering properties, Handbook of Set Theoretic Topology (K. Kunen and J. E. Vaughan, eds.), North-Holland, Amsterdam, 1984, pp. 347-422.

[GY] G. Gruenhage and Y. Yajima, A filter property of submetacompactness and its application to products, Topology Appl. 36 (1990), 43-55.

[I] F. Ishikawa, On countably paracompact spaces, Proc. Japan Acad. 31 (1955), 686-687.

[J] S. Jiang, On Junnila's problem, Questions Answers Gen. Topology 6 (1988), 43-47.

[Ju1] H. J. K. Junnila, Metacompactness, paracompactness and interior-preserving open covers, Trans. Amer. Math. Soc. 249 (1979), 373-385.

[Ju2] _- On submetacompactness, Topology Proc. 3 (1978), 375-405.

[K] Y. Katuta, Expandability and its generalizations, Fund. Math. 87 (1975), 231-250.

[M1] K. Morita, Paracompactness and product spaces, Fund. Math. 50 (1962), 223-236.

[M2] _ Note on paracompactness, Proc. Japan Acad. 37 (1961), 1-3.

[S] B. M. Scott, Toward a product theory of orthocompactness, Studies in Topology (N. M. Stavrakas and K. R. Allen, eds.), Academic Press, New York, 1975, pp. 517-537.

[T1] H. Tamano, On paracompactness, Pacific J. Math. 10 (1960), 1043-1047.

[T2] _ On compactifications, J. Math. Kyoto Univ. 1 (1962), 161-193.

[W] J. M. Worrell, Jr., A characterization of metacompact spaces, Portugal. Math. 25 (1966), 171-174.

Department of Mathematics, Kanagawa University, Yokohama 221, Japan 